Article

\title{
Essential Oil Analysis and Antimicrobial Evaluation of Three Aromatic Plant Species Growing in Saudi Arabia
}

\author{
Hamdi El-Said ${ }^{1}$, Sami S. Ashgar ${ }^{1}$, Ammar Bader ${ }^{2, *}$, Aljawharah AlQathama ${ }^{2}{ }^{\circ}$, Majed Halwani ${ }^{3}{ }^{(1)}$, \\ Roberta Ascrizzi 4 (D) and Guido Flamini ${ }^{4, *(D)}$ \\ 1 Department of Medical Microbiology, Faculty of Medicine, Umm Al-Qura University, Makkah 21955, \\ Saudi Arabia; hmibrahim@uqu.edu.sa (H.E.-S.); ssashgar70@hotmail.com (S.S.A.) \\ 2 Department of Pharmacognosy, Faculty of Pharmacy, Umm Al-Qura University, Makkah 21955, Saudi Arabia; \\ aaqathama@uqu.edu.sa \\ 3 King Abdullah International Medical Research Center, King Saud Bin Abdulaziz University for Health \\ Sciences, Riyadh 11481, Saudi Arabia; halawanima@ngha.med.sa \\ 4 Department of Pharmacy, University of Pisa, Via Bonanno 6, 56126 Pisa, Italy; roberta.ascrizzi@gmail.com \\ * Correspondence: ambader@uqu.edu.sa (A.B.); guido.flamini@unipi.it (G.F.)
}

Citation: El-Said, H.; Ashgar, S.S.; Bader, A.; AlQathama, A.; Halwani, M.; Ascrizzi, R.; Flamini, G. Essential Oil Analysis and Antimicrobial Evaluation of Three Aromatic Plant Species Growing in Saudi Arabia. Molecules 2021, 26, 959. https:// doi.org/10.3390/molecules26040959

Academic Editor: Henryk H. Jeleń

Received: 15 December 2020

Accepted: 8 February 2021

Published: 11 February 2021

Publisher's Note: MDPI stays neutral with regard to jurisdictional claims in published maps and institutional affiliations.

Copyright: (c) 2021 by the authors. Licensee MDPI, Basel, Switzerland. This article is an open access article distributed under the terms and conditions of the Creative Commons Attribution (CC BY) license (https:// creativecommons.org/licenses/by/ $4.0 /)$.

\begin{abstract}
Arabian flora is a rich source of bioactive compounds. In this study, we investigated three aromatic plant species with the aim of finding valuable sources of antimicrobial agents against common pathogenic microorganisms. We focused especially on microorganisms, which cause outbreaks of infectious disease during mass gatherings and pilgrimages season in Saudi Arabia. The essential oils of three aromatic plant species were hydrodistilled from flowering aerial parts of Lavandula pubescens Decne. and Pulicaria incisa subsp. candolleana E.Gamal-Eldin, and from leaves, stems, ripe and unripe fruits of Juniperus procera Hochst. Ex Endl. They were subsequently analyzed by gas chromatography-mass spectrometry (GC-MS). The main constituents of L. pubescens were found to be carvacrol (55.7\%), methyl carvacrol $(13.4 \%)$, and $\beta$-bisabolene $(9.1 \%)$. P. incisa subsp. Candolleana essential oil was rich in linalool (33.0\%), chrysanthenone (10.3\%), eugenol $(8.9 \%)$, and cis-chrysanthenol (8.0\%); the major components of J. procera essential oil were $\alpha$-pinene $(31.3-62.5 \%)$ and $\delta-3$-carene (7.3-30.3\%). These essential oils were tested against thirteen American Type Culture Collection (ATCC) strains of Gram-positive and Gram-negative bacteria using the agar diffusion assay. The only effective essential oil was that of L. pubescens and the most sensitive strains were Acinetobacter baumannii, Salmonella typhimurium, Shigella sonnei, Enterococcus faecalis and Staphylococcus epidermidis. Carvacrol, the major constituent of L. pubescens, was tested on these strains and was compared with vancomycin, amikacin, and ciprofloxacin. The Minimum Inhibitory Concentration (MIC) and Minimum Bactericidal Concentration (MBC) assays of L. pubescens essential oil and carvacrol revealed that Gram-negative strains were more susceptible than the Gram-positive ones.
\end{abstract}

Keywords: carvacrol; Lavandula pubescens; Pulicaria incisa; Juniperus procera; holy sites health

\section{Introduction}

Plants represent valuable sources of bioactive molecules belonging to various classes of secondary metabolites. Among the physiological roles of secondary metabolites in plants, is the resistance to phytopathogens, including bacteria, fungi and viruses [1-3]. The majority of these metabolites have the ability to interact with cellular enzymes or cell structure, causing irreversible damage to the invasive microorganisms [4-6]. For this reason, plant secondary metabolites have become an interesting target for the discovery of new bioactive molecules with antimicrobial effects and variable modes of action, especially after the recent emergence and growth of antibiotic resistance. According to the World Health Organization (WHO), the current rise in antibiotic resistance is due to the misuse of pharmaceutical antibiotics and is a major cause of the prolongation of illness with higher risk of death [7]. 
Essential oils (EOs) have numerous commercial applications due to their diverse biological properties and appealing fragrances. The global market thus supports a wide variety of pharmaceutical products such as gels, creams, ointments, nano-emulsions, and patches [8-11]. Besides their exceptional antimicrobial effects, they also possess a wide range of pharmacological properties, including, for example, analgesic, anti-inflammatory, antidiabetic, anti-parasitic, anticancer and antioxidant activity [12-16]. The use of EOs in therapy has increased in recent times due to the rise in multidrug-resistant bacteria and the high costs of new generation antibiotic drugs [17]. The chemical nature of essential oils hinders the process of microbial resistance, since they are very complex mixtures of constituents with different structures, including monoterpenes [18], sesquiterpenes [19], diterpenes [20], sulfur-containing compounds [21,22], phenylpropanoids [20], alkaloids [23], and phenols [24]. This large variety of chemical structures means that no single enzyme is able to deactivate all of these compounds. Thus, EOs may represent an open frontier for advances in medicine and pharmaceutical sciences.

The flora of Saudi Arabia is very rich and variable due to environmental diversity, ranging from extreme arid desert to high mountains with high rainfall rates. These variations affect plant metabolism in terms of secondary metabolite chemistry and biological activity. In fact, some Saudi plants have been found to exhibit substantial chemo-diversity from the same plants grown in other countries and climates [25-27]. The aim of this study was to investigate the chemical composition of hydrodistilled EOs from different organs of three aromatic plant species collected in Saudi Arabia and to find valuable sources of antimicrobial agents against common pathogenic microorganisms. These were the aerial parts of Lavandula pubescens Decne. and Pulicaria incisa subsp. candolleana E.Gamal-Eldin, as well as leaves, stems, ripe and unripe fruits of Juniperus procera Hochst. Ex Endl.

L. pubescens is reported in the literature as a species commonly found among the wild flora in Middle-Eastern Asia and Mediterranean Africa; it is, indeed, mainly reported as growing in Palestine [28], Yemen [29-31], Saudi Arabia [32-34]. For P. incisa subsp. Candolleana, to the best of our knowledge, only one study is reported in the literature, analyzing wild-growing specimens in Egypt [35]. However, Pulicaria spp. are reported as widely used in Northern African folk-medicine [36]. These species have been chosen for their good availability in the wild, which makes them an exploitable and easy to gather biomass in their native range.

Recently, in Saudi Arabia, new outbreaks of multidrug-resistant pathogenic microorganisms have been recorded in intensive care units, including Gram-positive and Gram-negative bacteria, out of which Acinetobacter baumannii, Pseudomonas aeruginosa, Escherichia coli, and Klebsiella pnemoniae can cause fatal respiratory tract infections and pneumonia, blood stream infections and urinary tract infections. Not only this, but also the transmission and spread of infectious diseases during mass public gatherings such as Hajj (an annual pilgrimage to the Holy Mecca, Saudi Arabia) poses an enormous challenge. The global spread of antibiotic-resistant bacteria by international travelers may occur during pilgrimages or when visitors return to their home countries [37]. The pathogenic microorganisms mentioned above have gradually become less susceptible to a broad spectrum of potent antibiotics such as imipenem, meropenem, ciprofloxacin, amikacin and cefuroxime [38]. Due to the increasing presence of multidrug-resistant pathogenic microorganisms, the present study also aims to test these three EOs against three Gram-positive and Gram-negative bacteria to assess their potential use as alternative antimicrobial agents.

\section{Results and Discussion}

\subsection{Essential Oil Compositions}

The six hydrodistilled EOs were analyzed by GC-MS; their complete compositions and hydrodistillation yields are reported in Table 1.

The EO of L. pubescens (Figure 1a) revealed a total of 19 different compounds. Oxygenated monoterpenes were the predominant class, accounting for $70.1 \%$ of the whole oil. Among them, carvacrol (55.7\%) and methyl carvacrol (13.4\%) were the main constituents. 
Monoterpene hydrocarbons were the second most abundant class, which included terpinolene $(6.1 \%),(Z)-\beta$-ocimene $(4.1 \%)$ and myrcene $(3.5 \%)$ among the most represented compounds. Sesquiterpene hydrocarbons accounted for $13.1 \%$, with $\beta$-bisabolene as the most abundant $(9.1 \%)$. The predominance of carvacrol and methyl carvacrol as the most abundant compounds in the L. pubescens EO of this study is in accordance with published compositions of the EOs hydrodistilled from several Yemeni specimens $[29,30]$. This species is, indeed, reported as having a phenolic-type EO profile among species belonging to this genus [39]. Several bioactivities of the EO are reported in the literature; for example, it is antioxidant and antimicrobial towards a wide variety of bacterial and fungal strains responsible for human and animal diseases, e.g., Staphylococcus aureus, E. coli, Candida albicans, and Microsporum canis $[28,30]$.

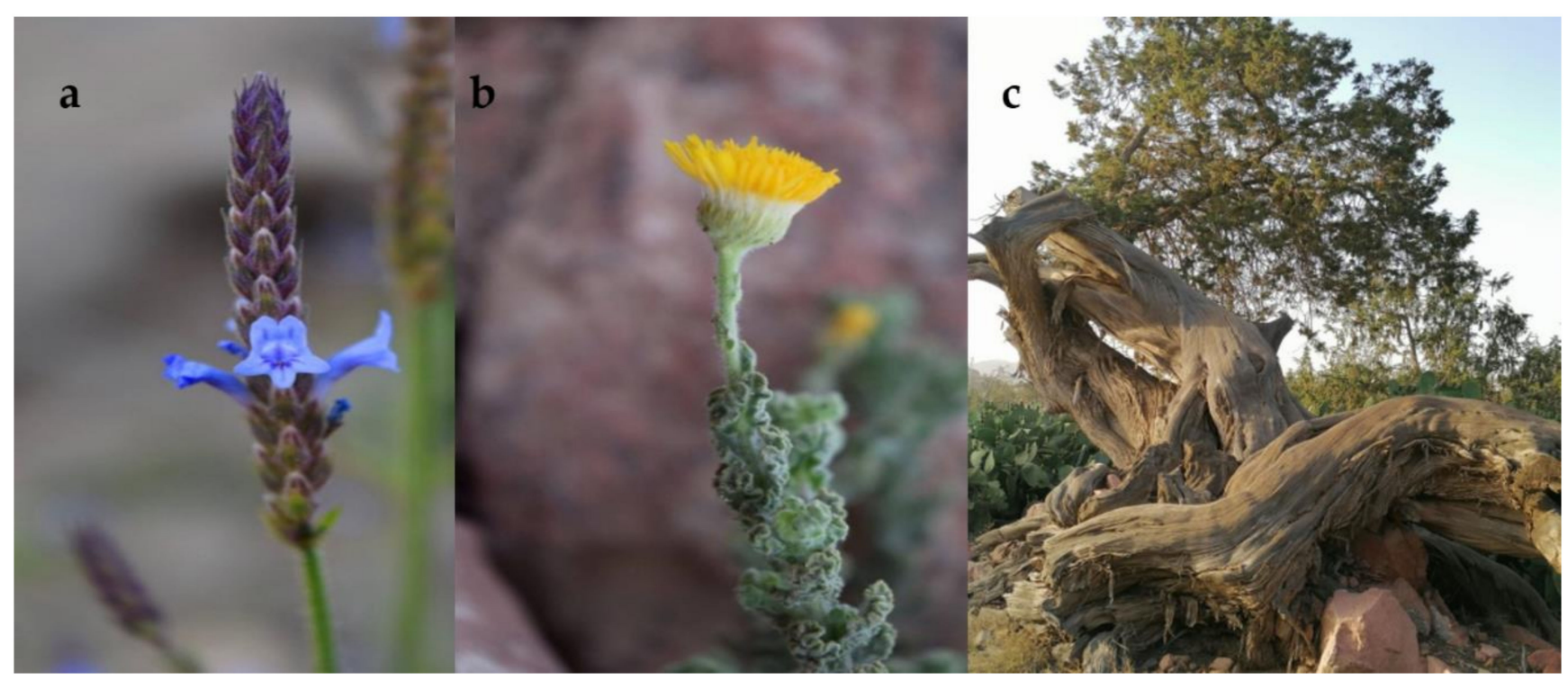

Figure 1. (a) Lavandula pubescens flowering aerial part; (b) Pulicaria incisa ssp. candolleana flowering aerial part; (c) Juniperus procera.

In the EOs of Pulicaria incisa ssp. candolleana (Figure 1b), 36 compounds were detected. Its $\mathrm{EO}$ was rich in oxygenated monoterpenes, accounting for $64.2 \%$ of the whole oil, with linalool (33.0\%), chrysanthenone $(10.3 \%)$, and cis-chrysanthenol $(8.0 \%)$ as the main constituents. Phenylpropanoids represented the second most abundant class, with eugenol $(8.9 \%)$ as the sole representative compound. The class of non-terpene derivatives accounted for $8.7 \%$, including mainly $(Z)$-jasmone (4.7\%) and isopentyl 2-methylbutanoate (2.6\%). Only one previous study reported the composition of the EOs of P. incisa ssp. candolleana collected in Egypt, with carvotanacetone (66.01-50.87\%) and chrysanthenone (13.26-24.3\%) as the most representative components, of which the latter was also present in a significant percentage $(10.3 \%)$ in our sample. EOs hydrodistilled from this Egyptian sample showed antimicrobial activity against Gram-positive and Gram-negative bacteria, as well as against some fungi, among which Streptococcus pneumonia, E. coli and Syncephalastrum racemosum were the most sensitive strains [35].

All EOs distilled from the different parts of J. procera (Figure 1c) were compared, revealing minor chemical differences. EO compositions showed some common compounds in all the investigated parts, such as $\alpha$-pinene (31.3-62.5\%), $\delta$-3-carene (7.3-30.3\%), $\alpha$-humulene (1.5-6.9\%), $\beta$-caryophyllene (1.6-6.4\%), and $\beta$-pinene (3.3-4.6\%). Ripe and unripe fruits showed the least differences, while the stems $\mathrm{EO}$ contained higher percentages of $\beta$-bisabolene $(9.1 \%)$, which was completely absent in leaf and fruit EOs. A previous study on the fruit $\mathrm{EO}$ of $J$. procera reported the presence of eugenol as the main constituent (78.4\%); although this phenylpropanoid was completely absent in our sample, these two compositions shared the presence of $\alpha$-pinene and $\beta$-caryophyllene, even in different percentages [40]. The cited study, however, does not specify the ripeness stage of the hy- 
drodistilled material. The EOs of the leaves reported in this study shared some constituents with Ethiopian samples, whose literature-reported EOs compositions showed $\alpha$-pinene (28.1\%), $\delta$-3-carene (29.6\%), $\beta$-pinene (4.35\%), elemol (1.8\%) and terpinolene $(4.1 \%)$ [41,42]. A published study by Burits et al. (2001) on J. Procera leaf EO reported its antioxidant capacity [43].

Table 1. Complete chemical composition of all the hydrodistilled essential oils.

\begin{tabular}{|c|c|c|c|c|c|c|c|c|}
\hline \multirow[t]{2}{*}{ Constituents. } & \multirow[t]{2}{*}{ 1.r.i. ${ }^{a}$} & \multirow[t]{2}{*}{ 1.r.i. $b$} & \multicolumn{6}{|c|}{ Relative Abundance (\%) } \\
\hline & & & L.pubescens & P. incisa & J.procera & J.procera & J.procera & J.procera \\
\hline & & & Aerial Parts & Aerial Parts & Leaves & Stems & Unripe Fruits & Ripe Fruits \\
\hline santolina triene & 910 & 908 & $-c$ & - & - & - & 0.1 & - \\
\hline tricyclene & 928 & 926 & - & - & - & 0.4 & 0.1 & - \\
\hline$\alpha$-thujene & 933 & 931 & - & 0.1 & - & 0.3 & - & - \\
\hline$\alpha$-pinene $*$ & 941 & 939 & - & 1 & 33.9 & 62.5 & 31.4 & 31.3 \\
\hline$\alpha$-fenchene & 954 & 951 & - & - & 1.6 & 0.5 & 1.2 & 1.1 \\
\hline camphene * & 955 & 953 & - & 0.2 & 0.6 & 0.7 & 0.3 & 0.5 \\
\hline thuja-2,4 (10)-diene & 959 & 957 & - & - & - & 0.3 & - & - \\
\hline sabinene * & 977 & 976 & - & - & - & 0.1 & 0.2 & - \\
\hline$\beta$-pinene $*$ & 982 & 980 & - & - & 4.6 & 3.4 & 3.6 & 3.3 \\
\hline 2,3-dehydro-1,8-cineole & 992 & 991 & - & 2.3 & - & - & - & - \\
\hline myrcene * & 993 & 991 & 3.5- & - & 3.7 & 3.3 & 4.1 & 4.2 \\
\hline cis-dehydroxylinalool oxide & 1002 & 999 & - & 0.2 & - & - & - & - \\
\hline$\alpha$-phellandrene ${ }^{*}$ & 1006 & 1005 & 0.2 & & & & & \\
\hline$\delta$-3-carene $*$ & 1013 & 1011 & 0.3 & - & 30.3 & 7.3 & 26.8 & 25.8 \\
\hline$\alpha$-terpinene ${ }^{*}$ & 1020 & 1018 & 0.2 & & & & & \\
\hline p-cymene * & 1028 & 1027 & 0.3 & 0.5 & 0.5 & 0.4 & 0.2 & 0.2 \\
\hline limonene * & 1032 & 1031 & 0.2 & 0.2 & 2.8 & 2.3 & 2.1 & 2.4 \\
\hline (Z)- $\beta$-ocimene * & 1042 & 1040 & 4.1 & - & - & - & - & - \\
\hline (E)- $\beta$-ocimene * & 1052 & 1050 & 0.4 & - & - & - & - & - \\
\hline$\gamma$-terpinene ${ }^{*}$ & 1063 & 1062 & - & 0.2 & - & - & 0.1 & 0.3 \\
\hline cis-linalool oxide (furanoid) * & 1076 & 1074 & - & 1.6 & - & - & - & - \\
\hline terpinolene * & 1089 & 1088 & 6.1 & - & 3.8 & 1.6 & 5.8 & 4.9 \\
\hline trans-linalool oxide (furanoid) * & 1090 & 1088 & - & 1.0 & - & - & - & - \\
\hline$p$-cymenene & 1091 & 1089 & - & - & - & 0.7 & - & - \\
\hline linalool * & 1101 & 1098 & - & 33.0 & 0.3 & 0.4 & 0.1 & - \\
\hline isopentyl-2-methylbutanoate * & 1102 & 1099 & - & 2.6 & - & - & - & - \\
\hline$\alpha$-cyclocitral & 1117 & 1116 & - & 0.4 & - & - & - & - \\
\hline$\alpha$-isophorone * & 1120 & 1118 & - & 0.3 & - & - & - & - \\
\hline chrysanthenone & 1126 & 1123 & - & 10.3 & - & - & - & - \\
\hline$\alpha$-campholenal & 1127 & 1125 & - & - & - & 0.6 & - & - \\
\hline trans-pinocarveol * & 1141 & 1139 & - & - & - & 1.1 & - & - \\
\hline camphor * & 1145 & 1144 & - & - & - & - & - & 0.2 \\
\hline trans-pinocamphone & 1162 & 1160 & - & - & - & 0.2 & - & - \\
\hline cis-chrysanthenol & 1163 & 1162 & - & 8.0 & - & - & - & - \\
\hline pinocarvone & 1164 & 1162 & - & - & - & 0.4 & - & - \\
\hline borneol * & 1168 & 1165 & - & - & - & 0.1 & - & - \\
\hline 4-terpineol * & 1179 & 1177 & - & 0.9 & - & 0.3 & - & - \\
\hline$p$-cymen-8-ol * & 1185 & 1183 & 0.7 & - & - & 0.2 & - & - \\
\hline$\alpha$-terpineol $*$ & 1191 & 1190 & 0.1 & 0.7 & 0.5 & 1.2 & 0.3 & 0.2 \\
\hline myrtenal * & 1194 & 1193 & - & - & - & 0.5 & - & - \\
\hline myrtenol * & 1195 & 1194 & - & - & - & 0.3 & - & - \\
\hline verbenone $*$ & 1207 & 1204 & - & 0.3 & - & 0.2 & - & - \\
\hline 8,9-dehydrothymol & 1221 & 1221 & - & 0.2 & - & - & - & - \\
\hline methylcarvacrol * & 1244 & 1244 & 13.4 & - & - & - & - & - \\
\hline cis-chrysanthenyl acetate & 1264 & 1262 & - & 1.3 & - & - & - & - \\
\hline isopiperitenone & 1271 & 1272 & - & 1.8 & - & - & - & - \\
\hline bornyl acetate * & 1287 & 1285 & - & - & 0.5 & 0.7 & 0.7 & 0.1 \\
\hline$p$-menth-1-en-9-ol & 1294 & 1291 & 0.2 & - & - & - & - & - \\
\hline carvacrol * & 1298 & 1298 & 55.7 & - & - & - & - & - \\
\hline eugenol * & 1358 & 1356 & - & 8.9 & - & - & - & - \\
\hline (E)- $\beta$-damascenone & 1382 & 1380 & - & 0.3 & - & - & - & - \\
\hline$\beta$-bourbonene & 1385 & 1384 & - & - & 2.0 & - & - & - \\
\hline$\beta$-elemene & 1392 & 1391 & - & - & 0.4 & - & 0.2 & 0.2 \\
\hline (E)-jasmone & 1393 & 1390 & - & 0.4 & - & - & - & - \\
\hline (Z)-jasmone * & 1395 & 1394 & - & 5.7 & - & - & - & - \\
\hline$\beta$-caryophyllene * & 1419 & 1418 & 3.8 & 1.1 & 2.9 & 1.6 & 5.9 & 6.4 \\
\hline dimethoxy-p-cymene & 1424 & 1423 & - & 0.2 & - & - & - & - \\
\hline$\alpha$-humulene * & 1455 & 1454 & 0.1 & - & 3.2 & 1.5 & 6.7 & 6.9 \\
\hline
\end{tabular}


Table 1. Cont.

\begin{tabular}{|c|c|c|c|c|c|c|c|c|}
\hline \multirow[t]{3}{*}{ Constituents. } & \multirow[t]{3}{*}{ 1.r.i. ${ }^{a}$} & \multirow[t]{3}{*}{ 1.r.i. ${ }^{b}$} & \multicolumn{6}{|c|}{ Relative Abundance (\%) } \\
\hline & & & L.pubescens & P. incisa & J.procera & J.procera & J.procera & J.procera \\
\hline & & & Aerial Parts & Aerial Parts & Leaves & Stems & Unripe Fruits & Ripe Fruits \\
\hline (E)- $\beta$-farnesene & 1459 & 1458 & - & - & - & 0.5 & - & - \\
\hline$\gamma$-muurolene & 1478 & 1477 & - & - & - & 0.4 & - & 0.1 \\
\hline germacrene D & 1482 & 1480 & - & - & 1.6 & 0.2 & 6.3 & 5.8 \\
\hline thymyl isobutyrate & 1490 & 1489 & - & 2.1 & - & - & - & - \\
\hline neryl isobutyrate * & 1492 & 1491 & - & 0.3 & - & - & - & - \\
\hline valencene * & 1493 & 1491 & - & - & - & 0.1 & - & - \\
\hline viridiflorene & 1495 & 1493 & - & - & - & - & - & 0.1 \\
\hline$\alpha$-muurolene & 1499 & 1499 & - & - & - & - & - & 0.1 \\
\hline germacrene $\mathrm{A}$ & 1505 & 1503 & - & - & - & - & - & - \\
\hline$\alpha$-bulnesene & 1507 & 1505 & - & - & - & - & 0.2 & 0.2 \\
\hline$\beta$-bisabolene & 1508 & 1509 & 9.1 & 2 & - & 9.1 & - & - \\
\hline trans- $\gamma$-cadinene & 1514 & 1513 & - & 0.7 & - & - & - & 0.2 \\
\hline$\delta$-cadinene & 1524 & 1524 & - & - & 0.2 & 0.4 & 0.3 & 0.4 \\
\hline elemol & 1550 & 1549 & - & - & 3.0 & 0.1 & 1.2 & 1.3 \\
\hline germacrene B & 1557 & 1556 & 0.1 & - & - & - & - & 0.3 \\
\hline germacrene-D-4-ol & 1574 & 1574 & - & - & - & - & 0.1 & 0.2 \\
\hline caryophyllene oxide * & 1582 & 1581 & 1.1 & 1.9 & 0.5 & 0.6 & - & 0.3 \\
\hline cedrol * & 1601 & 1599 & - & - & - & 1.7 & - & - \\
\hline humulene epoxide II & 1607 & 1606 & - & - & 0.4 & 0.5 & - & 0.3 \\
\hline 10-epi- $\gamma$-eudesmol & 1619 & 1619 & - & - & - & - & - & 0.2 \\
\hline$\gamma$-eudesmol & 1632 & 1630 & - & - & 0.5 & - & 0.1 & 0.3 \\
\hline T-cadinol & 1641 & 1640 & - & 1.4 & - & - & 0.1 & 0.3 \\
\hline$\beta$-eudesmol & 1650 & 1649 & - & 1.1 & - & - & 0.1 & 0.3 \\
\hline$\alpha$-cadinol & 1652 & 1653 & - & 0.3 & - & - & - & - \\
\hline$\alpha$-eudesmol & 1653 & 1652 & - & - & - & - & 0.4 & 1.2 \\
\hline Monoterpene-hydrocarbons & & & 15.3 & 2.2 & 81.8 & 83.8 & 76.0 & 74.0 \\
\hline Oxygenated-monoterpenes & & & 70.1 & 64.2 & 1.3 & 6.0 & 1.1 & 0.5 \\
\hline Sesquiterpene-hydrocarbons & & & 13.1 & 3.8 & 10.3 & 4.7 & 19.6 & 20.7 \\
\hline Oxygenated-sesquiterpenes & & & 1.2 & 4.7 & 4.4 & 2.9 & 2.0 & 4.4 \\
\hline Phenylpropanoids & & & - & 8.9 & - & - & - & - \\
\hline Apocarotenes & & & - & 1.0 & - & - & - & - \\
\hline Non-terpene-derivatives & & & - & 8.7 & - & - & - & - \\
\hline Total-identified (\%) & & & 99.6 & 93.5 & 97.8 & 97.4 & 98.7 & 99.6 \\
\hline Extraction yield $(\% w / w)$ & & & 1.09 & 1.14 & 0.51 & $<0.1$ & 2.7 & 2.39 \\
\hline
\end{tabular}

${ }^{a}$ Linear retentions index on a HP5-MS capillary column; ${ }^{b}$ values from the literature $[44,45]$; ${ }^{*}$ comparison with authentic standards; ${ }^{c}$ not detected.

\subsection{Antimicrobial Activity of the Essential Oils}

The EOs of L. pubescens, P. incisa ssp. candolleana, and J. procera leaves were tested against 13 different microbial strains, using diffusion assay on agar plates at the concentration of $20 \mu \mathrm{L}$ per well (equivalent to $200 \mu \mathrm{g})$; vancomycin, amikacin (30 $\mu \mathrm{g}$ per disc), and ciprofloxacin were used as positive controls (Table 2). Only the EO of L. pubescens was significantly active, and, among all tested strains, only towards Enterococcus faecalis ATCC 51299 (inhibition zone $12 \mathrm{~mm}$ ), Staphylococcus epidermidis ATCC 12228 (10 mm), Salmonella typhimurium ATCC 700720 (13 mm), A. baumannii (CRE) ATCC 1605 (15 mm), and Shigella sonnei ATCC 25931 (11 mm). A. baumannii has been reported by Haseeb et al. (2016) to be among the most common isolated Gram-negative pathogens, with high resistance rate to tobramycin, and E. faecalis and S. epidermidis are the most frequently reported Gram-negative pathogens during pilgrimage season [46]. In addition, antibiotic-resistant S. sonnei infections are commonly reported in mass gathering events [47]. Thus, finding antimicrobial activity in the EO of L. pubescens that works against these reported resistant pathogens will open the door to utilizing the essential oil of this plant as an alternative natural control against the spread of resistant pathogens during mass gatherings. 
Table 2. Antimicrobial evaluation of essential oils (EOs) in agar diffusion assay (200 $\mu \mathrm{g} /$ well), $\mathrm{R}=$ Resistant.

\begin{tabular}{cccc}
\hline Microbial Strains & $\begin{array}{c}\text { L. pubescens } \\
\text { Aerial Parts EO }\end{array}$ & $\begin{array}{c}\text { P. } \text { incisa } \\
\text { Aerial Parts EO }\end{array}$ & $\begin{array}{c}\text { J. procera } \\
\text { Leaves EO }\end{array}$ \\
\hline Enterococcusfaecalis ATCC * 51299 & $12 \mathrm{~mm}$ & $\mathrm{R}$ & $\mathrm{R}$ \\
\hline Enterococcusfaecalis(VRE) ATCC 51299 & $\mathrm{R}$ & $\mathrm{R}$ & $\mathrm{R}$ \\
\hline Staphylococcus aureus ATCC 25923 & $\mathrm{R}$ & $\mathrm{R}$ & $\mathrm{R}$ \\
\hline Staphylococcus aureus (MRSA) ATCC 43300 & $\mathrm{R}$ & $\mathrm{R}$ & $\mathrm{R}$ \\
\hline Staphylococcus epidermidis ATCC 12228 & $10 \mathrm{~mm}$ & $\mathrm{R}$ & $\mathrm{R}$ \\
\hline Salmonella typhimurium ATCC 700720 & $13 \mathrm{~mm}$ & $\mathrm{R}$ & $\mathrm{R}$ \\
\hline Klebsiella pneumonia (ESBL) ATCC 14028 & $\mathrm{R}$ & $\mathrm{R}$ & $\mathrm{R}$ \\
\hline Klebsiella pneumonia (CRE) ATCC 1705 & $\mathrm{R}$ & $\mathrm{R}$ & $\mathrm{R}$ \\
\hline Acinetobacter baumannii (CRE) ATCC 19605 & $15 \mathrm{~mm}$ & $\mathrm{R}$ & $\mathrm{R}$ \\
\hline Shigella sonnei ATCC 25931 & $11 \mathrm{~mm}$ & $\mathrm{R}$ & $\mathrm{R}$ \\
\hline Pseudomonas aeruginosa ATCC 15442 & $\mathrm{R}$ & $\mathrm{R}$ & $\mathrm{R}$ \\
\hline Proteus mirabilis ATCC 3071 & $\mathrm{R}$ & $\mathrm{R}$ & $\mathrm{R}$ \\
\hline Escherichia coli ATCC 35218 & $\mathrm{R}$ & $\mathrm{R}$ & $\mathrm{R}$ \\
\hline
\end{tabular}

* ATCC: American Type Culture Collection; VRE: Vancomycin-resistant Enterococci; MRSA: Methicillin-resistant Staphylococcus aureus; ESBL: Extended spectrum beta-lactamases; CRE: Carbapenem-resistant Enterobacteriaceae.

\subsection{Antimicrobial Activity of L. Pubescens and Carvacrol}

The EO of L. pubescens was tested at two concentrations $(200 \mu \mathrm{g}$ and $300 \mu \mathrm{g}$ per well) to obtain better information about its efficacy in comparison with its most abundant constituent (carvacrol). The EO exerted better inhibitory results against A. baumannii (CRE) ATCC 1605, compared to those obtained with carvacrol. This increased efficacy could be a synergistic effect of other constituents of L. pubescens EO. When testing carvacrol, inhibition zones were more pronounced than those of the crude oil for S. epidermidis ATCC $12228(15 \mathrm{~mm})$ and S. typhimurium ATCC $700720(20 \mathrm{~mm})$, A. baumannii (CRE) ATCC 1605 (15 mm), and S. sonnei ATCC 25931 (15 mm). Gram-positive strains were more sensitive

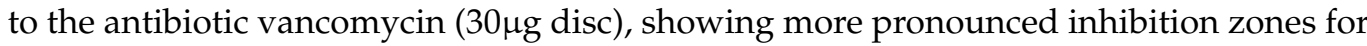
E. faecalis $(18.5 \mathrm{~mm})$ and S. epidermidis $(28 \mathrm{~mm})$.

In the case of Gram-negative strains, the antibiotic amikacin ( $30 \mu \mathrm{g}$ disc) showed inhibition activity against $S$. typhimurium $(20 \mathrm{~mm})$ and $A$. baumannii (CRE) $(15 \mathrm{~mm})$, while $S$. sonnei was observed to be resistant. All strains were not affected by dimethyl sulfoxide (DMSO), which was used as a negative control (Table 3). The antimicrobial activity of carvacrol has been reported similarly by others against E. faecalis [48], S. typhimurium [49], and $A$. baumannii indicating its potential effects in infectious disease control; antibacterial and antibiofilm activity against Salmonella enterica serotype; antimicrobial activity of essential oils-derived volatile compounds against several nosocomial pathogens including representative multidrug-resistant $A$. baumannii clinical isolates [9]. 
Table 3. Antimicrobial evaluation of carvacrol in dimethyl sulfoxide (DMSO) $10 \% w / v$ and L. pubescens EO in DMSO $10 \%$ $v / v$ in agar diffusion assay. $\mathrm{R}=$ Resistant. Data expressed as (mean $\pm \mathrm{SD}$ ) of two replicates.

\begin{tabular}{|c|c|c|c|c|c|c|c|}
\hline \multirow[b]{2}{*}{ Tested ATCC Strains } & \multicolumn{7}{|c|}{ Diameter of Zone of Inhibition (mm) } \\
\hline & $\begin{array}{l}\text { Carvacrol } \\
300 \mu \mathrm{g} / \text { well }\end{array}$ & $\begin{array}{l}\text { L. pubescens } \\
\text { EO } \\
200 \mu \mathrm{g} / \mathrm{well}\end{array}$ & $\begin{array}{c}\text { L. pubescens } \\
\text { EO } \\
300 \mu \mathrm{g} / \text { well }\end{array}$ & $\begin{array}{l}\text { Ciprofloxacin } \\
30 \mu \mathrm{g} / \text { well }\end{array}$ & $\begin{array}{l}\text { Amikacin } \\
30 \mu \mathrm{g} \text { (Disc) }\end{array}$ & $\begin{array}{l}\text { Vancomycin } \\
30 \mu \mathrm{g} \text { (Disc) }\end{array}$ & DMSO \\
\hline $\begin{array}{c}\text { Enterococcus faecalis } \\
\text { ATCC } 51299 \\
\end{array}$ & $12 \pm 0.00$ & $12 \pm 0.00$ & $14 \pm 0.00$ & - & - & $18.5 \pm 0.71$ & $\mathrm{R}$ \\
\hline $\begin{array}{c}\text { Staphylococcus epidermidis } \\
\text { ATCC } 12228 \\
\end{array}$ & $15 \pm 0.00$ & $10 \pm 0.00$ & $15 \pm 0.00$ & - & - & $28 \pm 0.00$ & $\mathrm{R}$ \\
\hline $\begin{array}{c}\text { Salmonella typhimurium } \\
\text { ATCC } 700720\end{array}$ & $20 \pm 0.00$ & $13 \pm 0.00$ & $19 \pm 0.00$ & $29 \pm 0.00$ & $20 \pm 0.00$ & - & $\mathrm{R}$ \\
\hline $\begin{array}{l}\text { Acinetobacter baumannii (CRE) } \\
\text { ATCC } 1605\end{array}$ & $15 \pm 0.00$ & $15 \pm 0.00$ & $24 \pm 0.00$ & $9 \pm 0.00$ & $15 \pm 0.00$ & - & $\mathrm{R}$ \\
\hline $\begin{array}{l}\text { Shigella sonnei } \\
\text { ATCC } 25931\end{array}$ & $15 \pm 0.00$ & $11 \pm 0.00$ & $16 \pm 0.00$ & $30 \pm 0.00$ & $\mathrm{R}$ & - & $\mathrm{R}$ \\
\hline
\end{tabular}

As shown in Table 4, Gram-negative strains exhibited a higher susceptibility to both EO and carvacrol than the Gram-positive ones (Table 4).

Table 4. Determination of the Minimum Inhibitory Concentration (MIC) and Minimum Bactericidal Concentration (MBC) expressed in $\mu \mathrm{g} / \mathrm{mL}$ of carvacrol, L. pubescens $\mathrm{EO}$, ciprofloxacin and vancomycin hydrochloride; data expressed as (mean $\pm \mathrm{SD}$ ) of two replicates.

\begin{tabular}{|c|c|c|c|c|c|c|c|c|}
\hline \multirow{2}{*}{ Tested ATCC Strains } & \multicolumn{2}{|c|}{ Carvacrol } & \multicolumn{2}{|c|}{ L. pubescens EO } & \multicolumn{2}{|c|}{ Ciprofloxacin } & \multicolumn{2}{|c|}{ Vancomycin } \\
\hline & MIC & MBC & MIC & MBC & MIC & MBC & MIC & MBC \\
\hline $\begin{array}{l}\text { Enterococcus faecalis } \\
\text { ATCC } 51299\end{array}$ & $500 \pm 0.00$ & $1000 \pm 0.00$ & $312 \pm 0.00$ & $625 \pm 0.00$ & - & - & $0.06 \pm 0.00$ & $0.12 \pm 0.00$ \\
\hline $\begin{array}{c}\text { Staphylococcus epidermidis } \\
\text { ATCC } 12228\end{array}$ & $500 \pm 0.00$ & $1000 \pm 0.00$ & $312 \pm 0.00$ & $625 \pm 0.00$ & - & - & $0.06 \pm 0.00$ & $0.12 \pm 0.00$ \\
\hline $\begin{array}{c}\text { Salmonella typhimurium } \\
\text { ATCC } 700720\end{array}$ & $250 \pm 0.00$ & $500 \pm 0.00$ & $78 \pm 0.00$ & $156 \pm 0.00$ & $0.70 \pm 0.17$ & $1.4 \pm 0.65$ & - & - \\
\hline $\begin{array}{l}\text { Acinetobacter baumannii } \\
\text { ATCC } 1605\end{array}$ & $250 \pm 0.00$ & $500 \pm 0.00$ & $78 \pm 0.00$ & $156 \pm 0.00$ & $15 \pm 0.00$ & $30 \pm 0.00$ & - & - \\
\hline $\begin{array}{l}\text { Shigella sonnei } \\
\text { ATCC } 25931\end{array}$ & $250 \pm 0.00$ & $500 \pm 0.00$ & $78 \pm 0.00$ & $156 \pm 0.00$ & $0.70 \pm 0.17$ & $1.4 \pm 0.65$ & - & - \\
\hline
\end{tabular}

As shown in Tables 3 and 4, both carvacrol and L. pubescens EO could be promising candidates for the development of formulas to be used mainly for the treatment of intestinal diseases caused by S. typhimurium and S. sonnei. The essential oil contact with these pathogens results in microorganism deactivation and a formulation of carvacrol with tetracycline hydrochloride was previous successfully used for the treatment of local mouth bacterial infections and candidiasis [8]. Further horizons could be established by the combinations of carvacrol or L. pubescens $\mathrm{EO}$ with classic antibiotics for the treatment of enteric pathogens.

\section{Materials and Methods}

\subsection{Plant Material}

Flowering aerial parts of Lavandula pubescens and different organs (leaves, stems, ripe and unripe fruits) of Juniperus procera were collected at Wadi Thee Ghazal, Near Taif, Makkah Province (GPS coordinates $21^{\circ} 05^{\prime} 56.1^{\prime \prime} \mathrm{N} 40^{\circ} 20^{\prime} 33.1^{\prime \prime} \mathrm{E}$ ), in June. Flowering aerial parts of Pulicaria incisa ssp. candolleana were collected at Jabal Al-Lawz, Tabuk province (GPS Coordinates $28^{\circ} 51^{\prime} 18.1^{\prime \prime} \mathrm{N}, 35^{\circ} 23^{\prime} 22.6^{\prime \prime} \mathrm{E}$ ), in November. Plants were photographed (Figure 1) and voucher specimens were deposited in the herbarium of the pharmacognosy lab, Umm Al-Qura University (L. pubescens, LP-EOM/SA-IT; J. procera JP-EOM/SA-IT; P. incisa ssp. candolleana PIC-EOM/SA-IT). 


\subsection{Chemicals and Reagents}

Solvents ( $n$-hexane HPLC grade, dimethyl sulfoxide (DMSO) analytical grade and carvacrol were purchased from Sigma-Aldrich (St. Louis, MO, USA). Mueller Hinton Agar was purchased from HiMedia Laboratories Pvt, Ltd. (Mumbai, India). Ciprofloxacin was purchased from Acros (New Jersey, USA), vancomycin and amikacin paper discs were purchased from Bioanalyse (Ankara, Turkey), vancomycin hydrochloride was kindly gifted by Hikma (Amman, Jordan).

\subsection{Essential Oil Extraction}

The air-dried plant material was finely crushed and subjected to EO hydrodistillation in a Clevenger-type apparatus for $2 \mathrm{~h}$. Aliquots of the obtained EOs were diluted to $10 \%$ in HPLC grade $n$-hexane prior to GC-MS injection, while the remaining parts were stored in freezer at $-18{ }^{\circ} \mathrm{C}$ until antimicrobial testing.

\subsection{Gas Chromatography-Mass Spectrometry Analyses and Peak Identification}

Gas chromatography-electron impact mass spectrometry (GC-EIMS) analyses were performed with an Agilent 7890B gas chromatograph (Agilent Technologies Inc., Santa Clara, CA, USA) equipped with an Agilent HP-5MS (Agilent Technologies Inc., Santa Clara, CA, USA) capillary column ( $30 \mathrm{~m} \times 0.25 \mathrm{~mm}$; coating thickness $0.25 \mu \mathrm{m})$ and an Agilent 5977B single quadrupole mass detector (Agilent Technologies Inc., Santa Clara, CA, USA). The oven temperature program was set to rise from $60{ }^{\circ} \mathrm{C}$ to $240{ }^{\circ} \mathrm{C}$ at $3{ }^{\circ} \mathrm{C} / \mathrm{min}$. Temperatures were set as follows: injector temperature, $220^{\circ} \mathrm{C}$; transfer-line temperature, $240{ }^{\circ} \mathrm{C}$. The carrier gas was He, at $1 \mathrm{~mL} / \mathrm{min}$ flow. The acquisition was performed with the following parameters: full scan, with a scan range of 35-300 m/z; scan time: $1.0 \mathrm{~s}$; threshold: 1 count. The identification of the constituents was based on the comparison of their retention times $\left(t_{R}\right)$ with those of pure reference samples and of their linear retention indices (LRIs), which were determined relatively to the $t_{R}$ of a series of $n$-alkanes (C9-C25). The detected mass spectra were compared with those listed in the commercial libraries NIST 14 and ADAMS, as well as in a homemade mass-spectral library, built up from pure substances and components of EOs of known composition and MS literature data [44,50].

\subsection{Diffusion Assay on Agar Plates}

As recommended by the National Committee for Clinical Laboratory Standards (NCCLS manual), the antimicrobial activity of EOs of the investigated plants was assayed by the diffusion method. The tested bacterial strains were: E. faecalis-ATCC 51299, E. faecalis (Vancomycin-resistant Enterococci, VRE), S. epidermidis-ATCC 12228, S. aureus -ATCC 43300, S. aureus (Methicillin-resistant Staphylococcus aureus, MRSA) 43300, S. typhimurium-ATCC 14028, A. baumannii, (Carbapenem-resistant Enterobacteriaceae, CRE)-ATCC 19605, S. sonnei-25931, K. pneumonia (Extended spectrum betalactamases, ESBL)_ATCC 700603, K. pneumonia (CRE)—ATCC 1705, P. aeruginosa, Proteus mirabilis-ATCC 43071, and E. coli-ATCC 35218. Above acronyms are as follows: ATTC: American Type Culture Collection; VRE: Vancomycin-resistant Enterococci; MRSA: Methicillin-resistant Staphylococcus aureus; ESBL: Extended spectrum beta-lactamases; CRE: Carbapenem-resistant Enterobacteriaceae. Each bacterial strain was suspended in Mueller-Hinton Broth and adjusted to 0.5 McFarland scale turbidity. The surface of MullerHinton agar plates was swabbed in three directions with standard inoculum, using sterile cotton swabs. The plates were allowed to dry for $10 \mathrm{~min}$ before 3-mm wells were cut into the Muller-Hinton agar using sterile plastic pipettes. Then, the wells were filled with $20 \mu \mathrm{L}$ and $30 \mu \mathrm{L}$ of EO dissolved in DMSO at the rate of $1 / 10 v / v$, since the EO of Lavandula pubescens was the only active one, the test was repeated by using the pure major constituent carvacrol at the concentration of $10 \mathrm{mg} / \mathrm{mL}$ in dimethyl sulfoxide (20 mg of carvacrol was dissolved in $2 \mathrm{~mL}$ of DMSO), $30 \mu \mathrm{L}$ per well (corresponding to $300 \mu \mathrm{g}$ per well), with vancomycin (30 $\mu \mathrm{g}$ disc) as a positive control for Gram-positive bacterial strains while amikacin (30 $\mu \mathrm{g}$ disc) and ciprofloxacin dissolved in DMSO with the rate 
$1000 \mu \mathrm{g} / 1000 \mu \mathrm{L}$ (30 $\mu \mathrm{g}$ per well) were used as a positive control for Gram-negative bacterial strains; furthermore, dimethyl sulfoxide was used as a negative control. All plates were incubated at $37^{\circ} \mathrm{C}$ for $24 \mathrm{~h}$ under aerobic conditions. After the incubation period, the plates were examined, and the diameter of each inhibition zone was measured.

\subsection{Microdilution of Broth Assay}

The microdilution method, using 96-well microtiter plates according to the Clinical and Laboratory Standards Institute (CLSI) guidelines [16], was conducted to evaluate the antibacterial activity. Performance standards for antimicrobial susceptibility testing were based on the 18th informational supplement of CLSI document Wayne (PA Clinical Laboratories Standards Institute, pp. 46-52) [9].

\subsection{Determination of the Minimum Inhibitory Concentration (MIC) and Minimum Bactericidal Concentration $(M B C)$}

MICs and MBCs of L. pubescens EO, carvacrol, ciprofloxacin, and vancomycin hydrochloride were determined with the broth micro-dilution method, with sterile 96-well microtiter plates for the determination of the MIC and MBCs of the tested samples. All samples were dissolved in DMSO, with exception of vancomycin hydrochloride which was dissolved in water. Basically, the first column in microtiter plates contained $200 \mu \mathrm{L}$ of EO, carvacrol, or antibiotics and the other subsequent wells contained $100 \mu \mathrm{L}$ of MuellerHinton broth. EO, carvacrol and antibiotic were serially diluted by transferring $100 \mu \mathrm{L}$ to the next well to produce serial dilutions. Mueller-Hinton broth $(100 \mu \mathrm{L}$ containing the bacteria $0.5 \mathrm{McF}$ arland) was added to each well containing $100 \mu \mathrm{L}$ of the tested EO, carvacrol or antibiotics. Sterilized Mueller-Hinton broth alone was used as the negative control, and bacterial broth with dimethyl sulfoxide (DMSO) was only used as control. The microdilution plates were incubated at $37^{\circ} \mathrm{C}$ overnight. The MIC was determined by selecting the lowest concentration of sample that completely inhibited the growth of the organism and compared with the growth control (Table 4). Wells with no visible growth in MIC were sub-cultured using $10 \mu \mathrm{L}$ of the selected wells and placed on Muller-Hinton agar plates. The MBC was determined by taking $10 \mu \mathrm{L}$ of the selected column and placing it on the Mueller-Hinton agar plates as well. All plates were incubated for $24 \mathrm{~h}$ at $37^{\circ} \mathrm{C}$ and the colony forming units (CFUs) were counted. MIC was determined by selecting the lowest concentration of the tested sample that completely inhibited the visible growth of a microorganism after overnight incubation in the well. The MBC was defined as the lowest concentration of the sample that prevents any growth of an organism after being sub-cultured on the Mueller-Hinton agar plate [9].

\section{Conclusions}

This study proposed the use of aromatic wild-growing species of Saudi Arabia as potential, natural sources of bioactive antimicrobial agents. As available and exploitable biomass, local wild-growing species can represent a promising source of new bioactive natural compounds, especially in the light of alternatives to traditional medicinal compounds, towards which antibiotic-resistance is a scary but growing phenomenon. Moreover, their availability and the facility of the proposed extraction method (hydrodistillation) constitute a remarkable cost reduction compared to existing antimicrobial agents.

L. pubescens and J. procera are abundantly distributed in the high region of Asir and Hijaz mountains, so they could be a source for mass production of essential oils, especially after the successful plantation in that region, while P. incisa subsp. candolleana belongs to an important genus, rich in essential oil with potential antimicrobial properties [51,52].

Our results encourage us to continue investigation into the possible mechanism of action of carvacrol, especially against $A$. baumannii, which is causing an increasing number of deaths in vulnerable patients. In addition, the EO of L. pubescens can be further studied for its use as an alternative natural approach to lowering the spread of infectious diseases during large gatherings and pilgrimages, such as Hajj. Furthermore, it could be of interest 
to verify a possible synergistic effect between the pure constituents of EOs and a number of antibiotics.

Author Contributions: Conceptualization, A.B.; methodology, A.B., G.F. and H.E.-S.; software, R.A., H.E.-S. and S.S.A.; validation, A.B. and G.F.; formal analysis, R.A., H.E.-S. and S.S.A.; investigation, R.A. and A.A.; resources, M.H; data curation, R.A.; writing-original draft preparation, A.B., A.A. and M.H.; writing-review and editing, G.F., R.A. and A.A.; supervision, A.B.; project administration, A.B.; funding acquisition, A.B. All authors have read and agreed to the published version of the manuscript.

Funding: The authors would like to thank the Deanship of Scientific Research at Umm Al-Qura University for supporting this work by Grant Code: (20UQU0055DSR).

Institutional Review Board Statement: Not applicable.

Informed Consent Statement: Not applicable.

Data Availability Statement: Data is contained within the article.

Conflicts of Interest: The authors declare no conflict of interest. The funders had no role in the design of the study; in the collection, analyses, or interpretation of data; in the writing of the manuscript, or in the decision to publish the results.

Sample Availability: Samples of the compounds are available from the authors.

\section{References}

1. Islam, W.; Qasim, M.; Noman, A.; Tayyab, M.; Chen, S.; Wang, L. Management of Tobacco Mosaic Virus through Natural Metabolites. Rec. Nat. Prod. 2018, 12, 403-415. [CrossRef]

2. Jiang, Z.; Jiang, H.; Xie, P. Antifungal activities against Sclerotinia sclerotiorum by Cinnamomum cassia oil and its main components. J. Essent. Oil Res. 2013, 25, 444-451. [CrossRef]

3. Della Pepa, T.; Elshafie, H.S.; Capasso, R.; De Feo, V.; Camele, I.; Nazzaro, F.; Scognamiglio, M.R.; Caputo, L. Antimicrobial and Phytotoxic Activity of Origanum heracleoticum and O. majorana Essential Oils Growing in Cilento (Southern Italy). Molecules 2019, 24, 2576. [CrossRef]

4. Yan, G.; Zhu, B.-R.; Tian, F.-L.; Hui, X.; Li, H.; Li, Y.-M.; Gao, W.-Y. Inhibitory Activity of Plant Essential Oils against E. coli 1-Deoxy-d-xylulose-5-phosphate reductoisomerase. Molecules 2019, 24, 2518. [CrossRef] [PubMed]

5. Soliman, S.; Alsaadi, A.; Youssef, E.; Khitrov, G.; Noreddin, A.; Husseiny, M.; Ibrahim, A. Calli Essential Oils Synergize with Lawsone against Multidrug Resistant Pathogens. Molecules 2017, 22, 2223. [CrossRef]

6. Oliva, A.; Costantini, S.; De Angelis, M.; Garzoli, S.; Božović, M.; Mascellino, M.; Vullo, V.; Ragno, R. High Potency of Melaleuca alternifolia Essential Oil against Multi-Drug Resistant Gram-Negative Bacteria and Methicillin-Resistant Staphylococcus aureus. Molecules 2018, 23, 2584. [CrossRef]

7. Ferri, M.; Ranucci, E.; Romagnoli, P.; Giaccone, V. Antimicrobial resistance: A global emerging threat to public health systems. Crit. Rev. Food Sci. Nutr. 2017, 57, 2857-2876. [CrossRef]

8. Obaidat, R.M.; Bader, A.; Al-Rajab, W.; Abu Sheikha, G.; Obaidat, A.A. Preparation of Mucoadhesive Oral Patches Containing Tetracycline Hydrochloride and Carvacrol for Treatment of Local Mouth Bacterial Infections and Candidiasis. Sci. Pharm. 2011, 79, 197-212. [CrossRef]

9. Omran, Z.; Bader, A.; Porta, A.; Vandamme, T.; Anton, N.; Alehaideb, Z.; El-Said, H.; Faidah, H.; Essa, A.; Vassallo, A.; et al. Evaluation of Antimicrobial Activity of Triphala Constituents and Nanoformulation. Evid.-Based Complement. Altern. Med. 2020, 2020, 6976973. [CrossRef] [PubMed]

10. Gemeda, N.; Tadele, A.; Lemma, H.; Girma, B.; Addis, G.; Tesfaye, B.; Abebe, A.; Gemechu, W.; Yirsaw, K.; Teka, F.; et al. Development, Characterization, and Evaluation of Novel Broad-Spectrum Antimicrobial Topical Formulations from Cymbopogon martini (Roxb.) W. Watson Essential Oil. Evid.-Based Complement. Altern. Med. 2018, 2018, 9812093. [CrossRef]

11. Komeh-Nkrumah, S.A.; Nanjundaiah, S.M.; Rajaiah, R.; Yu, H.; Moudgil, K.D. Topical Dermal Application of Essential Oils Attenuates the Severity of Adjuvant Arthritis in Lewis Rats. Phyther. Res. 2012, 26, 54-59. [CrossRef]

12. Amin, B.; Hosseinzadeh, H. Black Cumin (Nigella sativa) and Its Active Constituent, Thymoquinone: An Overview on the Analgesic and Anti-inflammatory Effects. Planta Med. 2015, 82, 8-16. [CrossRef]

13. Ali, B.H.; Blunden, G. Pharmacological and toxicological properties of Nigella sativa. Phyther. Res. 2003, 17, 299-305. [CrossRef]

14. Bedini, S.; Flamini, G.; Ascrizzi, R.; Venturi, F.; Ferroni, G.; Bader, A.; Girardi, J.; Conti, B. Essential oils sensory quality and their bioactivity against the mosquito Aedes albopictus. Sci. Rep. 2018, 8, 17857. [CrossRef] [PubMed]

15. Abdalla, A.N.; Shaheen, U.; Abdallah, Q.M.A.; Flamini, G.; Bkhaitan, M.M.; Abdelhady, M.I.S.; Ascrizzi, R.; Bader, A. Proapoptotic Activity of Achillea membranacea Essential Oil and Its Major Constituent 1,8-Cineole against A2780 Ovarian Cancer Cells. Molecules 2020, 25, 1582. [CrossRef]

16. Burits, M.; Bucar, F. Antioxidant activity of Nigella sativa essential oil. Phyther. Res. 2000, 14, 323-328. [CrossRef] 
17. Giuliani, A.; Pirri, G.; Nicoletto, S. Antimicrobial peptides: An overview of a promising class of therapeutics. Open Life Sci. 2007, 2, 1-33. [CrossRef]

18. Nostro, A.; Cannatelli, M.A.; Morelli, I.; Cioni, P.L.; Bader, A.; Marino, A.; Alonzo, V. Preservative properties of Calamintha officinalis essential oil with and without EDTA. Lett. Appl. Microbiol. 2002, 35, 385-389. [CrossRef]

19. Bader, A.; Caponi, C.; Cioni, P.L.; Flamini, G.; Morelli, I. Acorenone in the essential oil of flowering aerial parts of Seseli tortuosum L. Flavour Fragr. J. 2003, 18, 57-58. [CrossRef]

20. Bader, A.; Cioni, P.L.; De Tommasi, N.; Flamini, G. Essential Oil Compositions of Two Populations of Salvia samuelssonii Growing in Different Biogeographical Regions of Jordan. Nat. Prod. Commun. 2014, 9. [CrossRef]

21. Ascrizzi, R.; Flamini, G. Leek or Garlic? A Chemical Evaluation of Elephant Garlic Volatiles. Molecules 2020, $25,2082$. [CrossRef] [PubMed]

22. Bader, A.; Flamini, G.; Cioni, P.L.; Morelli, I. The Composition of the Root Oil of Salvadora persica L. J. Essent. Oil Res. 2002, 14, 128-129. [CrossRef]

23. Fico, G.; Bader, A.; Flamini, G.; Cioni, P.L.; Morelli, I. Essential Oil of Nigella damascena L. (Ranunculaceae) Seeds. J. Essent. Oil Res. 2003, 15, 57-58. [CrossRef]

24. Naik, D.G.; Dandge, C.N.; Rupanar, S.V. Chemical Examination and Evaluation of Antioxidant and Antimicrobial Activities of Essential Oil from Gymnema sylvestre R. Br. Leaves. J. Essent. Oil Res. 2011, 23, 12-19. [CrossRef]

25. Iannuzzi, A.M.; Camero, C.M.; D’Ambola, M.; D’Angelo, V.; Amira, S.; Bader, A.; Braca, A.; De Tommasi, N.; Germanò, M.P. Antiangiogenic Iridoids from Stachys ocymastrum and Premna resinosa. Planta Med. 2019, 85, 1034-1039. [CrossRef] [PubMed]

26. Bader, A.; Abdallah, Q.; Abdelhady, M.; De Tommasi, N.; Malafronte, N.; Shaheen, U.; Bkhaitan, M.; Cotugno, R. Cytotoxicity of Some Plants of the Asteraceae Family: Antiproliferative Activity of Psiadia punctulata Root Sesquiterpenes. Rec. Nat. Prod. 2019, 13, 307-315. [CrossRef]

27. Dal Piaz, F.; Bader, A.; Malafronte, N.; D’Ambola, M.; Petrone, A.M.; Porta, A.; Ben Hadda, T.; De Tommasi, N.; Bisio, A.; Severino, L. Phytochemistry of compounds isolated from the leaf-surface extract of Psiadia punctulata (DC.) Vatke growing in Saudi Arabia. Phytochemistry 2018, 155, 191-202. [CrossRef] [PubMed]

28. Ali-Shtayeh, M.S.; Abu-Zaitoun, S.Y.; Dudai, N.; Jamous, R.M. Downy Lavender Oil: A Promising Source of Antimicrobial, Antiobesity, and Anti-Alzheimer's Disease Agents. Evid.-Based Complement. Altern. Med. 2020, 2020, 5679408. [CrossRef] [PubMed]

29. Al-Badani, R.N.; Da Silva, J.K.R.; Setzer, W.N.; Awadh Ali, N.A.; Muharam, B.A.; Al-Fahad, A.J.A. Variations in Essential Oil Compositions of Lavandula pubescens (Lamiaceae) Aerial Parts Growing Wild in Yemen. Chem. Biodivers. 2017, 14, e1600286. [CrossRef]

30. Al-Badani, R.N.; Da Silva, J.K.R.; Mansi, I.; Muharam, B.A.; Setzer, W.N.; Awadh Ali, N.A. Chemical Composition and Biological Activity of Lavandula pubescens Essential Oil from Yemen. J. Essent. Oil Bear. Plants 2017, 20, 509-515. [CrossRef]

31. Shehata, I.A. Essential oils of Lavandula species growing in Yemen. Bull. Fac. Pharm. (Cairo Univ.) 2001, 39, $233-238$.

32. Albalawi, M.A.D.; Bashir, N.A.O.; Tawfik, A. Anticancer and Antifolate Activities of Extracts of Six Saudi Arabian Wild Plants Used in Folk Medicine. J. Life Sci. 2015, 10.

33. Hassan, S.A.; Al-Thobaiti, A.T.I. morphological nutlet characteristics of some lamiaceae taxa in Saudi Arabia and their taxonomic significance. Pak. J. Bot. 2015, 47, 1969-1977.

34. Alghamdi, S.B.; Abdelshafeek, K.A. Evaluation of Antimicrobial and Cytotoxic Activities of Different Extracts of Lavandula pubescens growing in Albaha Region, KSA. Int. J. Biol. Pharm. Allied Sci. 2019, 8, 1338-1353.

35. Shahat, E.A.; Bakr, R.O.; Eldahshan, O.A.; Ayoub, N.A. Chemical Composition and Biological Activities of the Essential Oil from Leaves and Flowers of Pulicaria incisa sub. candolleana (Family Asteraceae). Chem. Biodivers. 2017, 14, e1600156. [CrossRef]

36. El-Shahaby, O.; El-Zayat, M.; Rabei, R.; Aldesuquy, H.S. Phytochemical constituents, antioxidant activity and antimicrobial potential of Pulicaria incisa (lam.) DC as a folk medicinal plant. Prog. Chem. Biochem. Res. 2019, 2, $22-227$.

37. Leangapichart, T.; Gautret, P.; Griffiths, K.; Belhouchat, K.; Memish, Z.; Raoult, D.; Rolain, J.-M. Acquisition of a High Diversity of Bacteria during the Hajj Pilgrimage, Including Acinetobacter baumannii with bla OXA-72 and Escherichia coli with bla NDM-5 Carbapenemase Genes. Antimicrob. Agents Chemother. 2016, 60, 5942-5948. [CrossRef]

38. Al Johani, S.M.; Akhter, J.; Balkhy, H.; El-Saed, A.; Younan, M.; Memish, Z. Prevalence of antimicrobial resistance among gram-negative isolates in an adult intensive care unit at a tertiary care center in Saudi Arabia. Ann. Saudi Med. 2010, 30, 364-369. [CrossRef] [PubMed]

39. Aprotosoaie, A.C.; Gille, E.; Trifan, A.; Luca, V.S.; Miron, A. Essential oils of Lavandula genus: A systematic review of their chemistry. Phytochem. Rev. 2017, 16, 761-799. [CrossRef]

40. Almadiy, A.A. Chemical composition, insecticidal and biochemical effects of two plant oils and their major fractions against Aedes aegypti, the common vector of dengue fever. Heliyon 2020, 6, e04915. [CrossRef]

41. Adams, R.P. Juniperus procera of East Africa: Volatile leaf oil composition and putative relationship to J. excelsa. Biochem. Syst. Ecol. 1990, 18, 207-210. [CrossRef]

42. Adams, R.P. Systematics of multi-seeded eastern hemisphere Juniperus based on leaf essential oils and RAPD DNA fingerprinting. Biochem. Syst. Ecol. 1999, 27, 709-725. [CrossRef]

43. Burits, M.; Asres, K.; Bucar, F. The antioxidant activity of the essential oils of Artemisia afra, Artemisia abyssinica and Juniperus procera. Phyther. Res. 2001, 15, 103-108. [CrossRef]

44. Adams, R.P. Identification of Essential oil Components by Gas Chromatography/Quadrupole Mass Spectroscopy; Allured Publishing Corporation: Carol Stream, IL, USA, 1995; ISBN 1932633219. 
45. Linstrom, P.J.; Mallard, W.J. NIST Chemistry WebBook; NIST Standard Reference Database Number 69; National Institute of Standards and Technology: Gaithersburg MD, USA. [CrossRef]

46. Haseeb, A.; Faidah, H.S.; Bakhsh, A.R.; Al Malki, W.H.; Elrggal, M.E.; Saleem, F.; ur Rahman, S.; Khan, T.M.; Hassali, M.A Antimicrobial resistance among pilgrims: A retrospective study from two hospitals in Makkah, Saudi Arabia. Int. J. Infect. Dis. 2016, 47, 92-94. [CrossRef] [PubMed]

47. Al-Tawfiq, J.A.; Memish, Z.A. Potential risk for drug resistance globalization at the Hajj. Clin. Microbiol. Infect. 2015, 21, 109-114. [CrossRef]

48. Samadi, N.; Zaree, R.; Bakhtiar, H.; Salehnia, A.; Azimi, S. Comparative Antibacterial Efficacy of Endemic Satureja Khuzistanica Jamzad Essential Oil, Sodium Hypochlorite and Chlorhexidine Gluconate Solutions as Root Canal Irrigations. Dent. Res. J. (Isfahan) 2011, 8, 28-32.

49. Miladi, H.; Zmantar, T.; Kouidhi, B.; Chaabouni, Y.; Mahdouani, K.; Bakhrouf, A.; Chaieb, K. Use of carvacrol, thymol, and eugenol for biofilm eradication and resistance modifying susceptibility of Salmonella enterica serovar Typhimurium strains to nalidixic acid. Microb. Pathog. 2017, 104, 56-63. [CrossRef] [PubMed]

50. National Institute of Standards and Technology (NIST). NIST/EPA/NIH Mass Spectral Library; The NIST Mass Spectrometry Data Center: Gaithersburg, MD, USA, 2014.

51. Ansari, A.; Abbas, Z.; Saggu, S.; Rehman, H.; Moawed, M. Growth responses of Lavandula pubescens to temperature regimes of Tabuk, Saudi Arabia. J. Med. Plants Stud. 2014, 2, 38-41.

52. El-Juhany, L.; Aref, I.; Al-Ghamdi, M. Effects of Different Pretreatments on Seed Germination and Early Establishment of the Seedlings of Juniperus procera Trees. World Appl. Sci. J. 2009, 7, 616-624. 